\title{
'Transient' devices from silk, silicon and magnesium
}

A cross-disciplinary collaboration between Fiorenzo Omenetto, a biomedical engineer at Tufts University, Medford, MA, and John Rogers, a materials scientist at University of Illinois at Urbana-Champaign, has yielded tiny new electronic devices that completely dissolve when they are no longer needed (Science 337, 1640-1644; 2012). These 'physically transient' electronics are built from silicon, the preferred semiconductor, like other devices, but the silicon membranes in the transient devices are less than $100 \mathrm{~nm}$ thick. They are coated in silk packaging, processed from silkworm cocoons into thin, flexible films. Omenetto and Rogers can adjust the properties and thickness of the silicon and the silk to control the dissolution rates of the devices. The electrical components are made from magnesium, a metal whose reactivity largely excludes it from use in traditional electronics. In transient devices, however, its reactivity is a benefit. "Everything dissolves," Rogers told NPR's David Schultz (Shots, 27 September 2012; http://www.npr. org/blogs/health/2012/09/26/161815537/ medical-electronics-built-to-last-only-alittle-while).

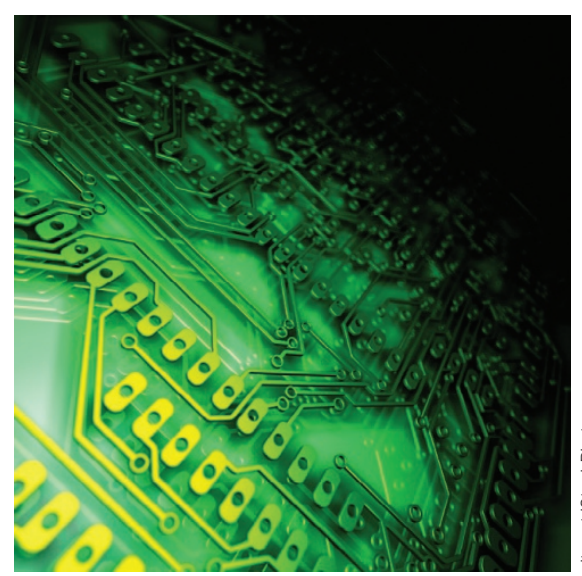

As a case example, Omenetto and Rogers built a dissolvable implant that heats up when hit with radio waves and tested it in rodents. By increasing local temperature, the device kills bacteria to prevent infection that might otherwise occur (after surgery, for example). The implants were well tolerated in BALB/c mice and Sprague-Dawley rats. Blood vessels grew into and around the silk, and there were no signs of inflammation where the devices had been placed. The devices dissolved almost completely in about 3 weeks.
There are several potential applications of this technology, including reducing the waste stream from consumer electronics. But the medical applications offer perhaps the biggest benefits. Dissolvable implants could eliminate the need for surgical removal, reducing potential complications, enhancing patient welfare and cutting health-care costs. Devices could be programmed to monitor internal physiology and release drugs accordingly or to produce electric current to help bones heal in situ.

It may be a decade or more before biodegradable devices are available for general use. Devices would need to be tested and approved by regulatory authorities, such as the US Food and Drug Administration, before they could be marketed. Large-scale manufacturing of the devices would also need to be addressed. Christopher Bettinger, who works with organic electronics at Carnegie Mellon University (Pittsburgh, PA) but was not involved in the study, suggests that the key to further development of this technology will be identifying a specific application-a disease or symptom that could be treated with such a device.

Monica Harrington

\section{REGENERATING RODENT DISCOVERED}

Certain species of amphibians and reptiles have defensive traits that allow them to escape predators, including the ability to lose and regenerate tissue, known as autotomy. Tissue regeneration is much more limited in mammals, however. Researchers have therefore depended on amphibian and reptile species to study wound healing, but their findings have been difficult to translate to humans due to the vast differences in biology between such species and mammals.

A new report describes two species of African spiny mouse (Acomys kempi and Acomys percivali) that can shed and regrow large portions of its skin, including hair follicles (Nature published online 27 September 2012; doi:10.1038/nature11499). Malcolm Maden (University of Florida, Gainesville) and collaborators at the University of Nairobi (Kenya) and Mpala Research Center (Nanyuki, Kenya) found that the skin of these mice easily tears upon handling, leading to large open wounds or skin loss that can total up to $60 \%$ of the animal's surface area. This likely developed as a defense mechanism that allows the mouse to release itself from a predator's grasp.

The team compared the mechanical properties of the skin of spiny mice and that of regular laboratory mice (Mus musculus). They found that Mus skin was 20 times stronger than Acomys skin, and nearly 77 times more energy was required to break it. Spiny mouse skin was also less elastic, probably because the large hair follicles and associated glands take up more space in the skin, reducing the overall amount of connective tissue. The spiny mice were able to heal their wounds much faster than the lab mice, restoring their skin and hair follicles within $3 \mathrm{~d}$. Additionally, spiny mouse skin did not scar. Unlike scar tissue, in which collagen fibers arrange into dense, organized bundles, the regenerating wounds maintained the looser collagen organization of undamaged tissue.

The researchers also tested whether the spiny mice could regenerate tissue to heal ear punch wounds, an ability that has been observed in rabbits but few other mammalian species. The mice were able to regenerate all tissue types, including skin, hair follicles, adipose cells and cartilage, except muscle.

In the future, the scientists plan to explore how the pathways regulating regeneration in the spiny mouse are modulated to produce tissue regrowth instead of scarring. One possibility is that the immune response is modulated to direct the healing process away from scar production toward tissue regeneration.

Kara Rosania 